\title{
Caracterização de treinadores de ciclismo da modalidade cross-country
}

\author{
Characterization of cross-country cycling coaches \\ Caracterización de los entrenadores de ciclismo de fondo
}

Recebido: 27/10/2021 | Revisado: 06/11/2021 | Aceito: 23/11/2021 | Publicado: 04/12/2021

Letícia Jaqueline Soares Cândido

ORCID: https://orcid.org/0000-0003-4092-2774

Faculdade Santa Rita, Brasil

E-mail: leticiacontato@yahoo.com.br

Lucas Rogério dos Reis Caldas

ORCID: https://orcid.org/0000-0002-7155-6319

Faculdade Santa Rita, Brasil

E-mail: lucasrrcaldas7@gmail.com

Luciano Bernardes Leite

ORCID: https://orcid.org/0000-0002-0885-5834 Universidade Federal de Viçosa, Brasil E-mail: luciano.leite@ufv.br

Leonardo Mateus Teixeira Rezende

ORCID: https://orcid.org/0000-0003-3942-3353 Instituto Federal de Educação, Ciência e Tecnologia Goiano, Brasil E-mail: leomtr.efi@gmail.com

Guilherme de Azambuja Pussieldi ORCID: https://orcid.org/0000-0002-0275-5226 Universidade Federal de Viçosa, Brasil E-mail: guipussi@ufv.br

Ricardo Luiz Pace Júnior

ORCID: https://orcid.org/0000-0003-0338-345X Faculdade Santa Rita, Brasil E-mail:ricardo_pace@yahoo.com.br

Josemara Fernandes Guedes de Sousa ORCID: https://orcid.org/0000-0003-0061-5729 Faculdade Santa Rita, Brasil

E-mail:josemaraf48@gmail.com

\begin{abstract}
Resumo
O estudo teve como objetivo avaliar o perfil de treinadores de ciclismo da modalidade cross-country. A amostra foi composta por 20 treinadores de ciclismo com idade entre 26 a 50 anos e que trabalhavam na prescrição de treinamento para ciclistas. Todos os voluntários responderam a um questionário semiestruturado, que abordou temas como: biografia dos treinadores; tempo de trabalho como treinador, forma que se mantém atualizado, tipos e formas de periodização, utilização de softwares para monitoramento dos atletas e os aspectos psicológicos do processo de treinamento. Os dados foram analisados de forma quantitativa através da distribuição de frequência absoluta e relativa. Observa-se que 55\% dos treinadores já trabalhavam a mais de 15 anos no ciclismo e 35\% da amostra também já trabalhavam a mais de 15 anos com atletas de alto rendimento. Também foi possível verificar que 100\% da amostra se mantém atualizada em relação aos seus conhecimentos relacionados a modalidade, sendo que $85 \%$ desses treinadores buscam esses conhecimentos através de cursos, congressos ou seminários científicos. Os principais resultados demonstraram que a maioria dos treinadores tinham pelo menos 15 anos de experiência na profissão, além disso todos os treinadores analisados afirmaram que se mantem atualizados em relação a literatura sobre a modalidade estudada.
\end{abstract}

Palavras-chave: Ciclismo; Cross country; Treinador.

\begin{abstract}
The study aimed to evaluate the profile of cross-country cycling coaches. The sample consisted of 20 cycling coaches aged between 26 and 50 years old and who worked on the training prescription for cyclists. All volunteers answered a semi-structured questionnaire that addressed topics such as: coaches' biography; working time as a coach, how to keep up to date, types and forms of periodization, use of software to monitor athletes and the psychological aspects of the training process. Data were analyzed quantitatively through the distribution of absolute and relative frequency. It is observed that $55 \%$ of the coaches had already worked for more than 15 years in cycling and $35 \%$ of the sample had also worked for more than 15 years with high-performance athletes. It was also possible to verify that $100 \%$ of the sample is kept up-to-date in relation to their knowledge related to the sport, with $85 \%$ of these coaches seeking this knowledge through courses, congresses or scientific seminars. The main results showed that most coaches had at least
\end{abstract}


15 years of experience in the profession, in addition, all coaches analyzed stated that they keep up-to-date in relation to the literature on the studied modality.

Keywords: Cycling; Cross country; Coach.

\begin{abstract}
Resumen
El estudio tuvo como objetivo evaluar el perfil de los entrenadores de ciclismo de fondo. La muestra estuvo formada por 20 entrenadores de ciclismo de entre 26 y 50 años que trabajaban en la prescripción de entrenamiento para ciclistas. Todos los voluntarios respondieron un cuestionario semiestructurado que abordó temas como: biografía de los entrenadores; tiempo de trabajo como entrenador, cómo mantenerse actualizado, tipos y formas de periodización, uso de software para monitorear a los atletas y los aspectos psicológicos del proceso de entrenamiento. Los datos se analizaron cuantitativamente mediante la distribución de frecuencia absoluta y relativa. Se observa que el $55 \%$ de los entrenadores ya había trabajado durante más de 15 años en el ciclismo y el 35\% de la muestra también había trabajado durante más de 15 años con deportistas de alto rendimiento. También se pudo comprobar que el 100\% de la muestra se mantiene actualizada en relación a sus conocimientos relacionados con el deporte, siendo el 85\% de estos entrenadores los que buscan estos conocimientos a través de cursos, congresos o seminarios científicos. Los principales resultados mostraron que la mayoría de los entrenadores tenían al menos 15 años de experiencia en la profesión, además, todos los entrenadores analizados manifestaron que se mantienen actualizados en relación a la literatura sobre la modalidad estudiada.
\end{abstract}

Palabras clave: Ciclismo; A campo traviesa; Entrenador.

\title{
1. Introdução
}

O mountain bike (MTB) é uma modalidade do ciclismo reconhecida pela Union Cyclist Internacionale (UCI), que é a associação internacional das federações nacionais de ciclismo, sem fins lucrativos e tem como função regulamentar o esporte, suas categorias e modalidades (Branco, 2018).

O MTB surgiu na década de 1970 e desde então tem atraído inúmeros praticantes e atletas em todo o mundo em diferentes faixas etárias (Sabeti-Aschraf et al., 2010), sendo que a modalidade se tornou popular a partir da inclusão nos jogos olímpicos de 1996 (Dingerkus et al., 1998; Kronisch; Pfeiffer, 2002). Esse esporte pode ser praticado em cenários naturais e com uma grande diversidade climática, onde o atleta é obrigado a passar por diversos obstáculos ao longo das corridas como terrenos escorregadios, acidentados e irregulares. Dessa forma, a prova exige um grande controle emocional dos atletas (Peinado et al., 2018).

O MTB é dividido em três tipos sendo eles, cross-country (XCO), montanha maratona de bicicleta (XCM) e o downhill (DHI) (Souza et al., 2016). O XCO é uma modalidade de resistência que nos últimos anos tem ganhado grande destaque no meio científico (Fornasiero et al., 2018). Ao longo dos anos vários estudos foram publicados sobre o esporte, dentre eles podemos destacar estudos sobre as demandas fisiológicas das competições e as características dos melhores ciclistas (Impellizzeri et al., 2002; Lee et al., 2002; Stapelfeldt et al., 2004; Impellizzer; Marcora, 2007;) além de estudos sobre o treinamento ideal para este tipo de desempenho no ciclismo (Costa, Nakamura, Oliveira, 2007; Inoue et al., 2016).

Apesar dos estudos sobre a modalidade citados acima, assim como em outros esportes pouco se sabe ainda sobre a importância e o perfil dos treinadores que acompanham e prescrevem treinamentos para os atletas. Os treinadores desempenham um papel importante sobre seus atletas (Forlenza et al., 2018; Viciana et al., 2015), influenciando principalmente no desenvolvimento das capacidades físicas e psicológicas (Potts et al., 2018; Forlenza et al. 2018). O treinador é responsável pela elaboração de métodos de treinamento para melhoria do desempenho individual e da equipe (GamonalesPuerto et al., 2020; Gómez-Cardona et al., 2019; Carson et al., 2019).

Além disso, segundo Balbino (2005) para ser treinador, além de possuir um conhecimento teórico, técnico e metodológico é necessário que os treinadores se mantenham sempre atualizados ou seja que eles acompanhem a evolução dos conhecimentos e da ciência no meio esportivo. O treinador para extrair o melhor desempenho do atleta treinado, deve buscar formas de monitorar a carga de treinamento do ciclista, levando em consideração carga interna e externa (Impellizzeri et al., 
2019; Inoue et al., 2021).

Além dos elementos citados acima, ser treinador de alto rendimento exige que o indivíduo tenha amplo conhecimento da modalidade, de aspectos físicos, biomecânicos e psicológicos que envolvem a modalidade de seu atleta. Muitas vezes esses treinadores não passam por uma educação formal para exercer a função, e buscam qualificar-se de forma autodirigida e através da experiência como treinadores para conseguirem extrair o melhor dos atletas por eles treinados. Essa situação, torna a profissão de treinador no alto rendimento esportivo um ambiente altamente exigente, imprevisível e inseguro (Bentzen et al., 2020; Impellizzeri et al., 2019; Inoue et al., 2021; Sherwin et al., 2016).

Portando, sabendo da importância dos treinadores no desempenho dos atletas, estudos que avaliem o perfil desses treinadores e seus métodos e formas de treinamento são de suma importância para o desenvolvimento do esporte. Todavia, no ciclismo se torna ainda mais importante estudos nesta temática, visto que como já mencionado anteriormente nos últimos anos o interesse pelo esporte tem crescido muito e o mesmo tem ganhado um grande destaque científico. Dessa forma o objetivo do presente estudo foi identificar o perfil de treinadores de ciclismo da modalidade cross-country.

\section{Metodologia}

\section{Tipo de estudo}

Este estudo é classificado quanto a natureza como uma pesquisa quantitativa, quanto ao objetivo trata-se de um estudo descritivo, quanto aos procedimentos caracteriza-se como um trabalho de levantamento. Uma vez que o mesmo utilizou de quantificações na coleta e tratamento dos dados, apresentando características de treinadores de ciclismo cross-country, através de um levantamento das características dos treinadores da modalidade (Leite \& Possa, 2013).

\section{Amostra}

A amostra foi composta por 20 treinadores de ciclismo com idade entre 26 a 50 anos e que trabalhavam na prescrição de treinamento para ciclistas profissionais da modalidade cross-country, todos os voluntários também eram formados em Educação Física. O estudo foi aprovado pelo Comitê de Ética e Pesquisa da Faculdade Santa Rita - CEP-FASAR, sendo aprovado sob número de parecer: 4.229.909, CAAE: 36125220.5.0000.8120, conforme parecer consubstanciado do CEPFASAR. Além da aprovação no comitê de ética, todos os voluntários tiveram que assinar um termo de Consentimento Livre e Esclarecido.

\section{Instrumentos e coleta de dados}

O instrumento utilizado foi um questionário semiestruturado, elaborado pelos próprios autores (Figura 1). O questionário é composto por 23 questões, que abordaram temas como: biografia dos treinadores; tempo de trabalho como treinador, forma que se mantém atualizado, tipos e formas de periodização, utilização de softwares para monitoramento dos atletas e os aspectos psicológicos do processo de treinamento. A duração média para responder o questionário foi de 20 minutos. O Link dos questionários foi enviado aos treinadores. Para coleta de dados foi utilizado os formulários do Google Forms. O Google Forms é uma ferramenta que permite a coleta de dados por meio de uma pesquisa personalizada, tipo Survey. Este método foi utilizado devido a situação atual do país que está em quarentena devido a pandemia da COVID-19. 
Figura 1 - Questionário sobre as estratégias utilizadas para treinamento de ciclismo modalidade Cross-country.

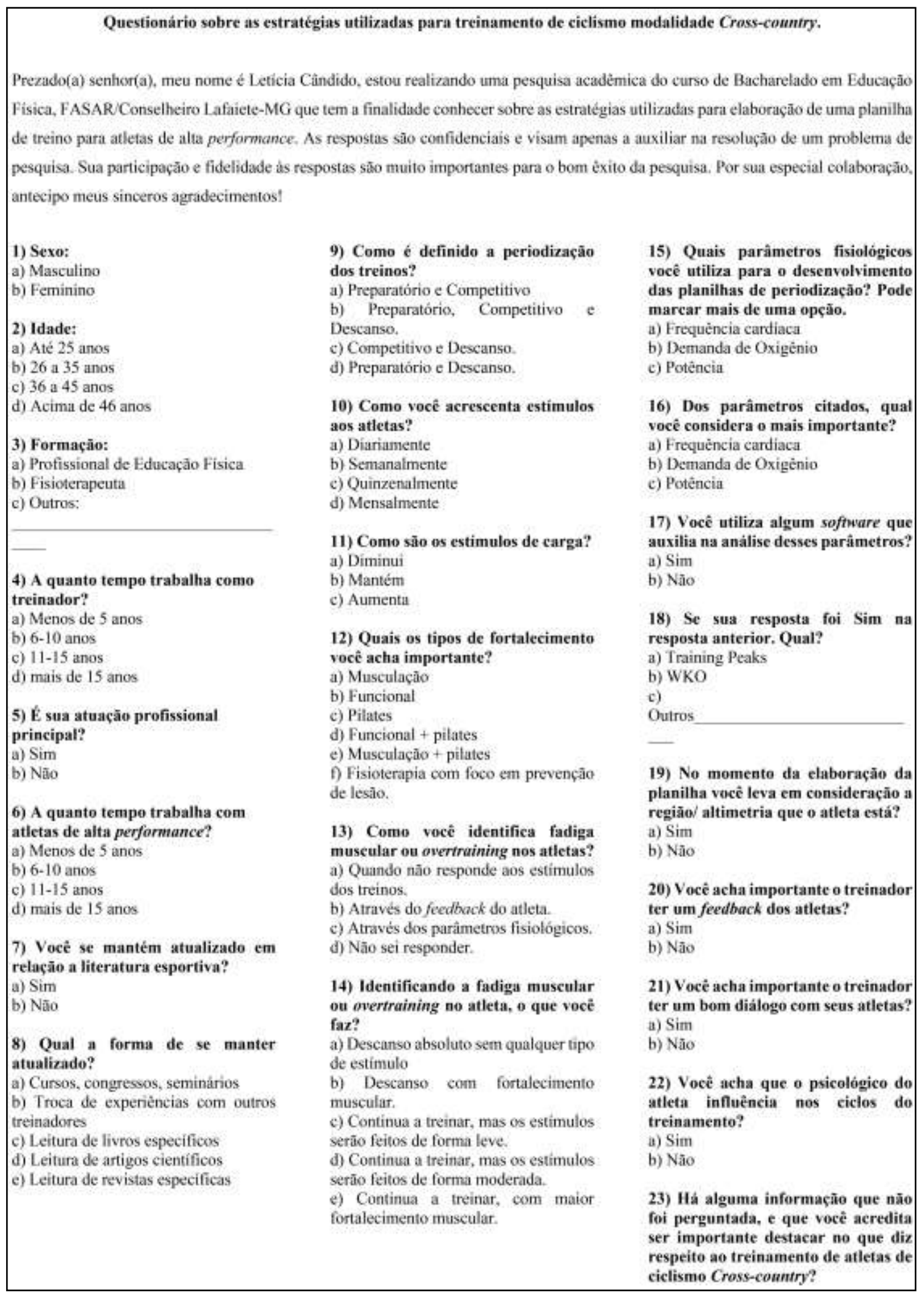

Fonte: Autores (2021). 


\section{Análise dos dados}

Os dados foram analisados de forma descritiva através da distribuição de frequência absoluta e relativa, para isso foi utilizado o pacote estatístico SPSS (Statistical Package for Social Sciences) versão 21.

\section{Resultados e Discussão}

A Tabela 1 apresenta os dados referentes a caracterização da amostra. Ao analisar os dados pode-se verificar que uma grande porcentagem de treinadores já trabalhava há mais de 15 anos (55\%) no ciclismo, demonstrando terem experiência na modalidade. Além disso, esses treinadores também já tinham uma elevada experiência com atletas, o que pode ser demonstrado pelas porcentagens de tempo de experiência, 6-10 anos (35\%), 11-15 anos (20\%) e mais de 15 anos (35\%). Nossos resultados vão de encontro a literatura, vários trabalhos tem mostrado que para um treinador ser considerado experiente em uma determinada modalidade ele deve ter em média 10 anos de experiência e no presente estudo encontramos grandes porcentagens de treinadores que possuíam esse tempo de experiência ou até mais na modalidade estudada (Côté et al, 1995; Schempp; Mccullick; Mason, 2006; Maduro, 2011). Esse resultado é importante, pois segundo Cunha et al., (2010) a experiência dos treinadores adquirida ao longo de suas carreiras, permitem um maior domínio dos atletas em relação aos processos de orientação, planejamento e gestão do treinamento a médio e longo prazo.

Tabela 1 - Caracterização da amostra.

\begin{tabular}{lcc}
\hline & $\mathrm{N}$ & $\%$ \\
\hline Sexo & 16 & $80,0 \%$ \\
Masculino & 4 & $20,0 \%$ \\
Feminino & & \\
Faixa Etária & 0 & $0,0 \%$ \\
Menos de 25 anos & 4 & $20,0 \%$ \\
$25-35$ anos & 15 & $75,0 \%$ \\
$36-45$ anos & 1 & $5,0 \%$ \\
Acima de 46 anos & & \\
Tempo de experiência como treinador & 1 & $5,0 \%$ \\
Menos de 5 anos & 6 & $30,0 \%$ \\
Entre $6-10$ anos & 2 & $10,0 \%$ \\
Entre $11-15$ anos & 11 & $55,0 \%$ \\
Mais de 15 anos & & $35,0 \%$ \\
Tempo de experiência com atletas & 7 & $10,0 \%$ \\
Menos de 5 anos & 7 & $35,0 \%$ \\
Entre $6-10$ anos & 7 & $20,0 \%$ \\
Entre $11-15$ anos & & \\
Mais de 15 anos & & \\
\hline
\end{tabular}

Fonte: Autores (2021).

A Tabela 2, apresenta os resultados referentes a atualização dos treinadores em seus conhecimentos e as formas como eles buscam esses conhecimentos do treinamento no ciclismo. Ao analisar os resultados pode-se verificar que $100 \%$ da amostra 
utiliza a literatura cientifica para se manter atualizado e $85 \%$ da amostra busca essa atualização dos conhecimentos por meio de cursos, congressos ou seminários científicos relacionados a modalidade estudada e de temas relacionados a mesma. Resultados semelhantes foram encontrados por Garcia (2018) que analisou o perfil de formação dos treinadores de esportes olímpicos que atuam com atletas e equipes de alto rendimento no Rio Grande do Sul e verificou que 100\% da amostra realizaram algum curso de formação específico de sua modalidade. Já em um outro estudo de Mendes et al., (2020) que avaliou as fontes de conhecimento de treinadores de handebol, foi possível verificar que as principais fontes de conhecimento dos treinadores foram, internet, cursos de curta duração, observação de outros treinadores e a experiência como atletas e etc. Dessa forma, segundo Moletta et al. (2019) entender as situações de aprendizagem dos treinadores é uma variável importante, pois fornece uma informação para as instituições, clubes, secretarias sobre a forma de aprendizagem mais utilizada dentre os treinadores e qual área apresenta um déficit, possibilitando assim que sejam desenvolvidos meios para uma formação continuada com o objetivo de atender as necessidades e interesses dos treinadores.

Tabela 2 - Atualização do conhecimento pelos treinadores de ciclismo da modalidade cross-country.

\begin{tabular}{lcc}
\hline & $\mathrm{N}$ & $\%$ \\
\hline Atualização em relação a literatura & 20 & $100 \%$ \\
Sim & 0 & $0,0 \%$ \\
Não & & \\
Forma de Atualização & 17 & $85,0 \%$ \\
Cursos, congressos, seminários & 2 & $10,0 \%$ \\
Troca de experiências com outros treinadores & 1 & $5,0 \%$ \\
Leitura de revistas específicas & 17 \\
\hline
\end{tabular}

Fonte: Autores (2021).

A Tabela 3 apresenta os dados referentes ao tipo e frequência da periodização utilizada pelos treinadores. Observa-se que $75 \%$ da amostra utiliza como base da periodização o período preparatório, competitivo e o descanso e planejam suas atividades semanalmente com seus atletas. Sabe-se que a periodização do treinamento é caracterizada como um método eficaz de planejamento e desenvolvimento de programas de treinamentos compostos por ciclos curtos ou longos com o objetivo principal de melhora da performance do atleta (Lorenz, Morrison, 2015; Issurin, 2010). Além disso, a periodização do treinamento pode proporcionar uma melhoria das capacidades motoras específicas (Issurin, 2010), prevenir leões (Rhea, et al, 2013; Stone, Stone, Sands, 2007) e desenvolver picos de desempenho (Issurin, 2010), portanto uma periodização bem organizada pode proporcionar melhores resultados esportivos. 
Tabela 3 - Tipo de Periodização e a Frequência dos Estímulos.

\begin{tabular}{lcc}
\hline & N & $\%$ \\
\hline Periodização & 15 & $75,0 \%$ \\
Preparatório, Competitivo e Descanso & 2 & $10,0 \%$ \\
Base, Preparatório, Competitivo e transição & 1 & $5,0 \%$ \\
Trabalho com blocos de treinos & 1 & $5,0 \%$ \\
Preparatório, específico, competitivo e transição & 1 & $5,0 \%$ \\
ATR & & \\
Frequência dos estímulos & 1 & $5,0 \%$ \\
Diariamente & 13 & $65,0 \%$ \\
Semanalmente & 1 & $5,0 \%$ \\
Quinzenalmente & 1 & $5,0 \%$ \\
Mensalmente & 4 & $20,0 \%$ \\
Outros & & \\
\hline
\end{tabular}

Fonte: Autores (2021).

A Tabela 4 apresenta os dados referentes aos tipos de treinamentos prescritos pelos treinadores para seus atletas, além da utilização de algum software para auxílio nos treinos. Uma grande porcentagem de treinadores utilizava como métodos de treinamento a musculação, o funcional e exercícios de fisioterapia com ênfase na prevenção de lesões. Já em relação a utilização ou não de software, todos os treinadores utilizavam algum programa no processo de monitoramento de seus atletas. Sabe-se que atualmente a tecnologia no meio esportivo tem como objetivo principal o desenvolvimento do atleta e a busca por uma melhora da performance esportiva (Coutinho, 2017). Portanto, é necessário que as tecnologias esportivas sejam incorporadas no dia a dia das organizações esportivas de uma forma sistematizada visando o desenvolvimento dos atletas para o futuro (Ringuet-Riot et al., 2013).

Tabela 4 - Tipos de Treinamentos realizados e a utilização de recursos tecnológicos.

\begin{tabular}{lcc}
\hline & $\mathrm{N}$ & $\%$ \\
\hline Treinamento físico utilizado & & \\
Musculação, Funcional, Fisioterapia & 15 & $75,0 \%$ \\
Funcional, Pilates, Fisioterapia & 2 & $10,0 \%$ \\
Musculação, Pilates & 2 & $10,0 \%$ \\
Musculação, Funcional, Pilates, Funcional, Fisioterapia & 1 & $5,0 \%$ \\
Utilização de software & & \\
Sim & 20 & $100 \%$ \\
Não & 0 & $0,0 \%$ \\
\hline
\end{tabular}

Fonte: Autores (2021).

A Tabela 5 apresenta os dados referentes a relação entre treinadores e atletas e a importância do acompanhamento psicológico dos atletas. Observa-se que $100 \%$ da amostra considera importante o feedback recebido pelos atletas após o treinamento, o diálogo com os atletas e a importância do acompanhamento psicológico dos seus atletas. Os resultados 
encontrados no presente estudo demonstram que os treinadores consideram importante o acompanhamento de questões referentes ao estado psicológico dos seus atletas e a relação com seus atletas no processo de acompanhamento e prescrição de seus treinos. Esse resultado é de suma importância pois segundo a Vasconcelos-Raposo (1991) os fatores psicológicos são determinantes para o rendimento esportivo, sendo que a dimensão psicológica é responsável por 40 a $90 \%$ do sucesso esportivo. Um dos exemplos mais clássicos da literatura é a firmação do nadados Mark Spitz, que relatou que $99 \%$ do seu sucesso nos Jogos Olímpicos foi da sua preparação mental e não física ou técnica (Vasconcelos-Raposo, 1991; Williams, 1993). Além disso, sabe-se que um desequilíbrio emocional pode levar a depressão, o que consequentemente levam os atletas ao adoecimento. Segundo De Jesus Frades et al., (2020) a depressão no esporte é causada por diversos fatores, dentre eles podemos citar: personalidade do atleta, ansiedade, baixa autoestima, fracasso, lesão física, mudanças de comportamento, autocobrança, problemas afetivos, perda de prestígio ou da posição de titular, baixo rendimento, dentre outras causas. No mesmo estudo, é citado diversos casos de atletas que sofreram depressão ao longo de suas carreiras, como, Anthony Ervin e Michael Phelps, nadadores americanos, Diego Hypólito, ginasta brasileiro, Allison Schmitt, nadador brasileiro, Rafaela Silva, judoca brasileira.

Tabela 5 - Acompanhamento psicológico dos atletas.

\begin{tabular}{lcc}
\hline & $\mathrm{N}$ & $\%$ \\
\hline Importância feedback dos atletas & 20 & $100 \%$ \\
Sim & 0 & $0,0 \%$ \\
Não & & \\
Importância diálogo com os atletas & 20 & $100 \%$ \\
Sim & 0 & $0,0 \%$ \\
Não & & \\
Importância psicológico & 20 & $100 \%$ \\
Sim & 0 & $0,0 \%$ \\
Não & & \\
\hline
\end{tabular}

Fonte: Autores (2021).

\section{Conclusão}

Este estudo teve como objetivo identificar o perfil de treinadores de ciclismo da modalidade cross-country. Os resultados do presente estudo indicam que a maioria dos treinadores eram do sexo masculino, a faixa etária predominante foi de 36 a 45 anos, a maior parte dos treinadores tinham pelo menos 15 anos de experiência na profissão e trabalhavam com atletas de alto rendimento há pelo menos 6 anos. Todos os treinadores afirmaram atualizar seus conhecimentos na literatura, sendo que as principais fontes declaradas foram cursos, congressos e seminários. O tipo de periodização mais utilizado foi preparatório, competitivo e descanso, sendo que os estímulos eram atualizados pela maioria dos treinadores semanalmente. Os tipos de treinamentos complementares mais utilizados foram musculação, treinamento funcional e fisioterapia. Todos os treinadores relataram fazer uso de softwares para monitoramento e controle do treinamento e consideravam o feedback ao atleta e um bom diálogo com os mesmos importantes, além de destacarem a importância do trabalho de um psicólogo para otimizar o rendimento dos atletas.

Até onde é de nosso conhecimento, este é um dos primeiros estudos que visou identificar o perfil de treinadores de ciclismo cross-country no Brasil. Pesquisas futuras podem complementar o perfil de treinadores da modalidade, identificando 
tempo de prática, nível de competição e podiums alcançados pelos atletas que são treinados pelos treinadores, quantidade e grau de importância das competições que os treinadores já participaram, análise das áreas científicas que os treinadores se fundamentam para atualização profissional, tipo e distribuição da prática para o treinamento de diferentes valências físicas, entre outros fatores que podem ajudar na compreensão do tema.

\section{Referências}

Bentzen, M., Kenttä, G., Richter, A., \& Lemyre, P. N. (2020). Impact of Job Insecurity on Psychological Well- and Ill-Being among High Performance Coaches. International journal of environmental research and public health, 17(19), 6939. https://doi.org/10.3390/ijerph17196939

Branco, G. R. (2018). Fatores musculoesqueléticos e de posicionamento sobre a bicicleta associados à ocorrência de dor anterior no joelho em ciclistas de mountain bike. Dissertação (Mestrado) - Programa de Pós-Graduação em Reabilitação e Desempenho Funcional, Universidade Federal dos Vales do Jequitinhonha e Mucuri, Diamantina.

Carson, F., Malakellis, M., Walsh, J., Main, L. C., \& Kremer, P. (2019). Examining the Mental Well-Being of Australian Sport Coaches. International journal of environmental research and public health, 16(23), 4601. https://doi.org/10.3390/ijerph16234601

Coté, J., Saimela, J., Trudel, P., Baria, A., \& Russell, S. (1995). The coaching model: A grounded assessment of expert gymnastic coaches' knowledge. Journal of sport and exercise psychology, 17(1), 1-17.

Coutinho, L. D. C. S. (2017). Gestão da Tecnologia e Inovação no Esporte: Estudo de Caso do Voleibol Brasileiro. Dissertação (Mestrado) - Faculdade de Desporto da Universidade do Porto, Porto - Portugal.

Cunha, G. B. D., Mesquita, I. M. R., Rosado, A. F. B., Sousa, T., \& Pereira, P. (2010). Necessidades de formaçao para o exercício profissional na perspectiva do treinador de futebol em funçao da sua experiencia e nível de formaçao. Motriz: Revista de Educação Física, 16, 931-941. https://doi.org/10.5016/1980$6574.2010 \mathrm{v} 16 \mathrm{n} 4 \mathrm{p} 931$

de Jesus Frades, L., Oliveira, E. S. A., Varoli, B. M., \& Carneiro, C. D. M. C. (2020). Adoecimento psíquico em atletas de alto rendimento: a importância da psicologia do esporte. Revista Educação, Psicologia e Interfaces, 4(3), 1-16. https://doi.org/10.37444/issn-2594-5343.v4i3.297

Dingerkus, M. L., Martinek, V., Kolzow, I., \& Imhoff, A. (1998). Mountainbike related injuries and overuse syndromes. Dtsch Z Sportmed, $49(8), 242-4$.

Forlenza, S. T., Pierce, S., Vealey, R. S., \& Mackersie, J. (2018). Coaching behaviors that enhance confidence in athletes and teams. International Sport Coaching Journal, 5(3), 205-212. https://doi.org/10.1123/iscj.2017-0040

Fornasiero, A., Savoldelli, A., Modena, R., Boccia, G., Pellegrini, B., \& Schena, F. (2018). Physiological and anthropometric characteristics of top-level youth cross-country cyclists. Journal of sports sciences, 36(8), 901-906. https://doi.org/10.1080/02640414.2017.1346271

Garcia, G. D. S. (2018). Um estudo sobre a formação de treinadores de esporte de alto rendimento de modalidades olímpicas no Rio Grande do Sul. Dissertação (Mestrado) - Programa de Pós-Graduação em Ciências do Movimento Humano da Escola de Educação Física, Fisioterapia e Dança da Universidade Federal do Rio Grande do Sul, Porto Alegre.

Gómez-Carmona, C. D., Gamonales-Puerto, J. M., Feu, S., \& Ibáñez, S. J. (2019). Study of internal and external load by different instruments. A case study in grassroots. Sport Sci J Sch Sport Phys Educ Psychomot, 5(3), 444-468. https://doi.org/10.17979/sportis.2019.5.3.5464

Impellizzeri, F., Sassi, A., Rodriguez-Alonso, M., Mognoni, P., \& Marcora, S. (2002). Exercise intensity during off-road cycling competitions. Medicine and science in sports and exercise, 34(11), 1808-1813. https://doi.org/10.1097/00005768-200211000-00018

Impellizzeri, F. M., \& Marcora, S. M. (2007). The physiology of mountain biking. Sports medicine (Auckland, N.Z.), 37(1), 59-71. https://doi.org/10.2165/00007256-200737010-00005

Impellizzeri, F. M., Marcora, S. M., \& Coutts, A. J. (2019). Internal and External Training Load: 15 Years On. International journal of sports physiology and performance, 14(2), 270-273. https://doi.org/10.1123/ijspp.2018-0935

Inoue, A., do Carmo, E. C., de Souza Terra, B., Moraes, B. R., Lattari, E., \& Borin, J. P. (2021). Comparison of coach-athlete perceptions on internal and external training loads in trained cyclists. European journal of sport science, 1-7. Advance online publication. https://doi.org/10.1080/17461391.2021.1927198

Inoue, A., Impellizzeri, F. M., Pires, F. O., Pompeu, F. A., Deslandes, A. C., \& Santos, T. M. (2016). Effects of Sprint versus High-Intensity Aerobic Interval Training on Cross-Country Mountain Biking Performance: A Randomized Controlled Trial. PloS one, 11(1), e0145298. https://doi.org/10.1371/journal.pone.0145298

Issurin V. B. (2010). New horizons for the methodology and physiology of training periodization. Sports medicine (Auckland, N.Z.), 40(3), 189-206. https://doi.org/10.2165/11319770-000000000-00000

Kronisch, R. L., \& Pfeiffer, R. P. (2002). Mountain biking injuries: an update. Sports medicine (Auckland, N.Z.), 32(8), 523-537. https://doi.org/10.2165/00007256-200232080-00004.

Lee, H., Martin, D. T., Anson, J. M., Grundy, D., \& Hahn, A. G. (2002). Physiological characteristics of successful mountain bikers and professional road cyclists. Journal of sports sciences, 20(12), 1001-1008. https://doi.org/10.1080/026404102321011760

Leite, F. C. L.; \& Possa, A. D. (2013). Metodologia da pesquisa científica. 2. ed. rev. Florianópolis : IFSC. 
Research, Society and Development, v. 10, n. 16, e02101622383, 2021

(CC BY 4.0) | ISSN 2525-3409 | DOI: http://dx.doi.org/10.33448/rsd-v10i16.22383

Lorenz, D., \& Morrison, S. (2015). Current concepts in periodization of strength and conditioning for the sports physical therapist. International journal of sports physical therapy, 10(6), 734. PMC4637911

Maduro, L. A. R. (2012). A Formação e a sua influência no papel do treinador de Judô no planeamento dos treinos e nas competições. Dissertação (Doutorado). Faculdade de Desporto - Universidade do Porto, Porto-Portugal.

Moletta, A. F., Mendes, F. G., de Angeloni Borges, L., \& Galatti, L. R. (2019). Treinadores e treinadoras de basquetebol de Santa Catarina: o desenvolvimento da aprendizagem formal, informal e não-formal. e-balonmano. com: Revista de Ciencias del Deporte, 15(3), 197-206. ISSN 1885 - 7019

Peinado, A. B., Romero-Parra, N., Rojo-Tirado, M. A., Cupeiro, R., Butragueño, J., Castro, E. A., Calderón, F. J., \& Benito, P. J. (2018). Physiological Profile of an Uphill Time Trial in Elite Cyclists. International journal of sports physiology and performance, 13(3), 268-273. https://doi.org/10.1123/ijspp.2016-0768

Potts, A. J., Didymus, F. F., \& Kaiseler, M. (2019). Exploring stressors and coping among volunteer, part-time and full-time sports coaches. Qualitative Research in Sport, Exercise and Health, 11(1), 46-68. https://doi.org/10.1080/2159676X.2018.1457562

Puerto, J. M. G., Carmona, C. D. G., León, K., Jiménez, J. M., \& Godoy, S. J. I. (2020). Estudio de las variables pedagógicas en tareas de entrenamiento en fútbol-base según el mesociclo competitivo: Un estudio de casos. Retos: nuevas tendencias en educación física, deporte y recreación, (37), 486-492. ISSN: $1579-1726$

Rhea, M. R., Ball, S. D., Phillips, W. T., \& Burkett, L. N. (2002). A comparison of linear and daily undulating periodized programs with equated volume and intensity for strength. The Journal of strength \& conditioning research, 16(2), 250-255.

Ringuet-Riot, C. J., Hahn, A., \& James, D. A. (2013). A structured approach for technology innovation in sport. Sports Technology, 6(3), 137-149. https://doi.org/10.1080/19346182.2013.868468

Sabeti-Aschraf, M., Serek, M., Geisler, M., Schmidt, M. I., Pachtner, T., Ochsner, A., ... \& Graf, A. (2010). Overuse Injuries Correlated to the Mountain Bikes Adjustment: A Prospective Field Study. The Open Sports Sciences Journal, 3(1): 1-6. ISSN: 1875-399X/10

Schempp, P. G., McCullick, B., \& Mason, I. S. (2006). The development of expert coaching. In The sports coach as educator (pp. 163-179). Routledge. ISBN: 9780203020074

Sherwin, I., Campbell, M. J., \& Macintyre, T. E. (2017). Talent development of high performance coaches in team sports in Ireland. European journal of sport science, 17(3), 271-278. https://doi.org/10.1080/17461391.2016.1227378

Souza, F. J., Santos, A. G. C., de Morais, K. A., Mendes, C. R. D. S., \& de Oliveira, V. M. (2016). Análise do Perfi 1 dos Praticantes de Mountain Bike (MTB) da Cidade de Trindade (GO). Vita et Sanitas, 10(1), 22-37.

Stapelfeldt, B., Schwirtz, A., Schumacher, Y. O., \& Hillebrecht, M. (2004). Workload demands in mountain bike racing. International journal of sports medicine, 25(4), 294-300. https://doi.org/10.1055/s-2004-819937

Stone, M. H., Stone, M., \& Sands, W. A. (2007). Principles and practice of resistance training. Human Kinetics.

Vasconcelos-Raposo, J. (1991). Contributo para a identificação do estado ideal para a prestação desportiva. Natação, 14(4), 4-8.

Viciana, J., Mayorga-Vega, D., Ruiz, J., \& Blanco, H. (2016). The educational communication delivered by soccer coaches in competition. RETOS-Neuvas Tendencias en Educacion Fisica, Deporte y Recreacion, (29), 17-21. ISSN: 1579-1726

Williams, J. M. E. (1993). Applied sport psychology: Personal growth to peak performance. Mayfield Publishing Co. 